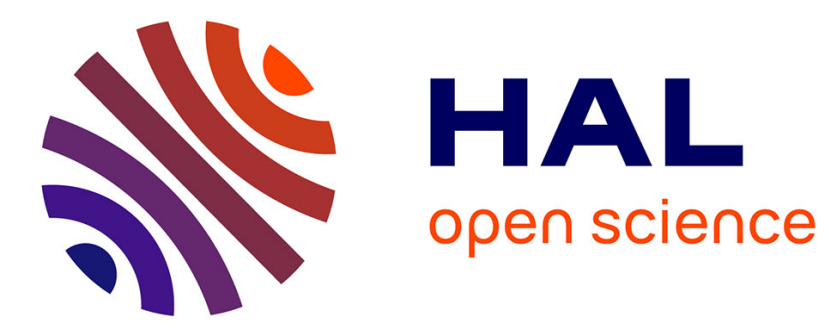

\title{
Dislocations in plastically deformed SrTiO3
}

Wilfried Sigle, Corneliu Sarbu, Dieter Brunner, Manfred Ruhle

\section{To cite this version:}

Wilfried Sigle, Corneliu Sarbu, Dieter Brunner, Manfred Ruhle. Dislocations in plastically deformed SrTiO3. Philosophical Magazine, 2006, 86 (29-31), pp.4809-4821. 10.1080/14786430600672695 . hal00513688

\section{HAL Id: hal-00513688 \\ https://hal.science/hal-00513688}

Submitted on 1 Sep 2010

HAL is a multi-disciplinary open access archive for the deposit and dissemination of scientific research documents, whether they are published or not. The documents may come from teaching and research institutions in France or abroad, or from public or private research centers.
L'archive ouverte pluridisciplinaire HAL, est destinée au dépôt et à la diffusion de documents scientifiques de niveau recherche, publiés ou non, émanant des établissements d'enseignement et de recherche français ou étrangers, des laboratoires publics ou privés. 


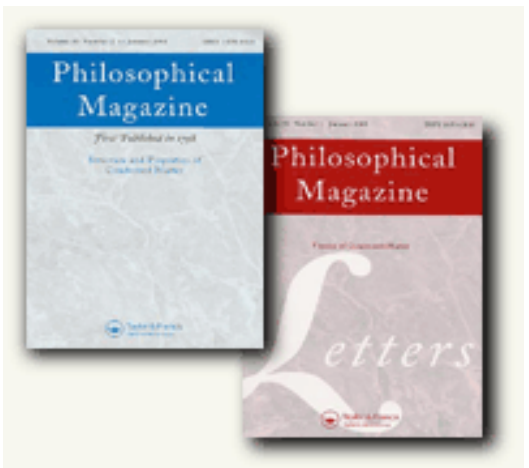

\section{Dislocations in plastically deformed SrTiO3}

\begin{tabular}{|r|l|}
\hline Journal: & Philosophical Magazine \& Philosophical Magazine Letters \\
\hline Manuscript ID: & TPHM-05-Dec-0538.R1 \\
\hline Journal Selection: & Philosophical Magazine \\
\hline Date Submitted by the & 01 -Mar-2006 \\
\hline Complete List of Authors: & $\begin{array}{l}\text { Sigle, Wilfried; Max-Planck-Institut für Metallforschung } \\
\text { Sarbu, Corneliu; National Institute for Materials Physics } \\
\text { Brunner, Dieter; Max-Planck-Institut für Metallforschung } \\
\text { Ruhle, Manfred; Max-Planck Institut, Metallforschung }\end{array}$ \\
\hline Keywords: & $\begin{array}{l}\text { transmission electron microscopy, brittle-ductile transition, } \\
\text { perovskites, plasticity of crystals, dislocation structures }\end{array}$ \\
\hline Keywords (user supplied): & \multicolumn{2}{|c}{} \\
\hline \multicolumn{2}{|c}{} \\
\hline
\end{tabular}

\section{\) ScholaroNE" \\ Manuscript Central}




\title{
Dislocations in plastically deformed $\mathrm{SrTiO}_{3}$
}

\author{
W. SIGLE, C. SARBU ${ }^{1)}$, D. BRUNNER, and M. RÜHLE \\ Max-Planck-Institut für Metallforschung, Heisenbergstraße 3, D-70569 Stuttgart \\ ${ }^{1)}$ On leave from: National Institute for Materials Physics, Magurele-Bucharest, Romania
}




\begin{abstract}
Bright-field and weak-beam TEM investigations were performed on plastically deformed $\mathrm{SrTiO}_{3}$. A gradual change from $a<110>$ edge-type to screw-type dislocations is found with decreasing temperature. It was found that edge dislocations undergo climb at high temperatures. In order to explain this plastic behaviour a model is presented which is based on the temperature dependence of the dislocation core structures. At elevated temperatures edge dislocations dissociate by climb. This is impeded at low temperatures because of the lack of thermal activation. At low temperatures screw dislocations are assumed to become sessile, probably due to a non-planar dissociation of the dislocation core.
\end{abstract}

Keywords: Strontium titanate; Plasticity; Dislocation; Brittle-to-ductile transition 


\section{Introduction}

Strontium titanate is an oxide ceramic with perovskite structure. It is cubic except below 105 $\mathrm{K}$ where the crystal structure changes to tetragonal and below $65 \mathrm{~K}$ to orthorhombic $(c / a=$ 1.002). Despite this seemingly simple structure the plastic behaviour (under compression) of this material recently turned out to exhibit quite a spectacular ductile-to-brittle-to-ductile transition (DBDT) [1,2] (see Fig.1).

[Insert Fig.1 about here]

At high temperatures (Regime C) the material yields at stresses which strongly depend on temperature. In this temperature regime flow stresses are considerably lower for compression along other directions than $<100>$ because then the easy glide system $a<100>\{100\}$ is active [3]. Towards lower temperatures, the material becomes brittle (Regime B), the transition from Regime $\mathrm{C}$ to Regime $\mathrm{B}$ occurring within a very narrow temperature range. Within Regime $\mathrm{B}$ the material fails by brittle fracture at a temperature-dependent fracture stress. Most surprisingly, the material becomes ductile again below about $1000 \mathrm{~K}$ (Regime A). The flow stress is low (about $150 \mathrm{MPa}$ ) and does not vary much until about ambient temperature. At lower temperatures the flow stress increases again. This increase is smooth except around 200 $\mathrm{K}$ where the flow stress shows a step-like behaviour (not shown in Fig.1) [4]. Note that this temperature is well above the cubic/tetragonal phase transition and is therefore not related with a structural change of the material.

Notwithstanding the orientation-dependent magnitudes of flow stresses in Regime $\mathrm{C}$ there is a general trend of this plastic behaviour irrespective of the crystallographic direction of compression. Slip planes and Burgers vectors were determined by optical polarized light microscopy and transmission electron microscopy (TEM), respectively [3]. For compression along $\langle 100\rangle$, dislocations with Burgers vector $a<110>$ (in the following called ' $a<110>$ dislocations') are predominant in the whole temperature range. For compression along directions different from $<100>, a<110>$ dislocations are present in Regime A, whereas the slip vector is $a<100>$ in Regime C.

Because the crystal structure does not change above $105 \mathrm{~K}$ it was supposed that this yield behaviour must be related to intrinsic effects of the perovskite material [2]. More precisely, it 
was argued that temperature-induced variations of the dislocation core structure might be responsible. In a series of papers we analysed the structures and chemical composition of different dislocation cores: $a<100>\{011\}$ edge [5], $a<110>\{110\}$ edge [6], and $a<100>$ screw [7]. These were dislocations in bi-crystals containing low-angle grain boundaries, i.e. not dislocations formed during plastic deformation. The bi-crystals were diffusion-bonded at 1700 $\mathrm{K}$, which is within temperature regime $\mathrm{C}$. It was found that the $a<100>\{011\}$ edge dislocation possesses a compact core structure with a widening of the core of only one lattice constant. On the contrary, the $a<110>\{110\}$ edge dislocation exhibits a climb dissociation into two $a / 2<110>$ partial edge dislocations separated by a $2.5 \mathrm{~nm}$ wide anti-phase boundary on a $\{110\}$ plane. The $a<100>$ screw dislocation does not show dissociation into separate partial dislocations but detailed analysis of atom positions shows an anisotropic widening of the core along $<110>$ directions. Using electron energy-loss spectroscopy, for all dislocations an increase of the Ti-to-O ratio was found in the core region suggesting a charged core. For the $a<110>\{110\}$ edge dislocation the presence of charge was indeed confirmed by impedance spectroscopy measurements on the same bi-crystals [8].

In the present paper we will present weak-beam and conventional bright-field images of dislocations in single-crystalline specimens deformed within Regimes A and C. These provide new insight into the yield mechanisms.

\section{Experimental}

Nominally undoped $\mathrm{SrTiO}_{3}$ crystals (30 - 200 at.ppm Ca, Ba, and $\mathrm{Mg}$ were found by atomic emission spectroscopy) were deformed in compression (for details see [3]). In the present paper we only discuss specimens compressed parallel to the [001] direction. TEM specimens were prepared by ion milling (for details see [3]). The specimens were cut parallel to the principle slip plane (011). The slip planes were selected from light-optical micrographs using polarised light. Since the specimens studied here were compressed along a $<100>$ axis, the crystals were cut at $45^{\circ}$ with respect to the compression axis. TEM studies were performed in 200 kV microscopes (Philips CM200, JEOL JEM2000FX). Weak-beam images were obtained using $(\boldsymbol{g}, 3 \ldots 4 \boldsymbol{g})$ conditions. The analyses involved large tilt angles in order to obtain 3dimensional information of the dislocation configuration.

\section{Results}




\subsection{Regime C, $1700 \mathrm{~K}$}

The typical dislocation arrangement after deformation in Regime $\mathrm{C}$ is shown in Fig. 2 (see also Fig.20 in [3]). It is a view approximately along the [233] axis using [0 $\overline{1} 1]$ as diffraction vector.

[Insert Fig. 2 about here]

There are predominantly straight edge dislocations from top left to bottom right. In addition, there are curved dislocations (vertical and from bottom left to top right) which have screw components. In Fig. 20 of Ref. [3] also pure screw dislocations were visible in this temperature regime. All dislocations have Burgers vectors of the $a<110>$-type, but they belong to different slip systems. This is because under compression along $<100>$ there exist four equivalent $a<110>\{110\}$ slip systems of which one is usually dominant because of slight misalignments during compression. The straight dislocations from top left to bottom right have $a[01 \overline{1}]$ Burgers vector and remain straight during tilt around the $[01 \overline{1}]$ axis. A second micrograph of this area is shown in Fig.3.

[Insert Fig. 3 about here]

For this image the crystal was tilted by $52^{\circ}$ close to the [001] zone axis using [110] as diffraction vector. This tilt includes a major tilt $\left(\approx 45^{\circ}\right)$ around the [100] line direction of the straight edge dislocations. Obviously the dislocation lines appear curved after this tilt. For clarity some corresponding dislocations are marked by letters.

\subsection{Regime A, $115 \mathrm{~K}$ and $\mathrm{RT}$}

Weak-beam dark-field images of dislocation configurations after deformation at room temperature and $115 \mathrm{~K}$ are shown in Figs. 4 and 5, respectively.

[Insert Figs. 4, 5 about here]

The diffraction vector $\boldsymbol{g}$ is $[0 \overline{1} 1]$. The Burgers vectors of all dislocations are $a[0 \overline{1} 1]$. Regarding the line directions, after $115 \mathrm{~K}$ deformation dislocations are predominantly of 
screw type whereas this is much less pronounced for room temperature deformation. Only few pure edge dislocations are visible, some of which are remarkably straight. Most of the dislocations show double contrast. Images taken under $+\boldsymbol{g}$ - and $\boldsymbol{- g}$-conditions show pronounced inside/outside contrast (Figs.6a,b) indicating that the double contrast is caused by dislocation dipoles.

[Insert Fig. 6a,b about here]

The double contrast is not always continuous along the dislocation line. At some places double contrast is visible at $+\boldsymbol{g}$, whereas at other places $\boldsymbol{- g}$ creates double contrast (marked by white arrows in Fig.6). Despite considerable effort (large tilt experiments) no indication of dislocation dissociation could be found.

Some of the screw dislocations showed a variation of the contrast along their dislocation line (Fig.7), being out of contrast for short segments of the line one of which is marked by an asterisk. The visible parts appeared to be slightly inclined with respect to the general direction of the dislocation line.

[Insert Fig. 7 about here]

\section{Discussion}

Although all types of $a<110>$ dislocations (from edge to screw) are found in the various temperature regimes, there is a clear trend from edge-type at high temperatures towards screw-type at low temperatures. We therefore believe that the slower moving species are of edge-type at high temperatures and of screw-type at low temperatures.

\subsection{Regime C}

As shown in [3] the dislocations observed in Regime C are $a<110>$ edge dislocations. These glide on either $(0 \overline{1} \overline{1}),(1 \overline{1} \overline{1})$, or (111) planes. Their straight appearance at small tilt angles (view perpendicular to the slip plane) shows that glide of $a<110>$ edge dislocations on the (011) slip plane appears to be inhibited. The most likely reason for this is that the dislocations are dissociated by climb. This phenomenon has been frequently observed in oxide ceramics 
[Insert Fig. 9 about here]

One of the partials is located in the centre $(x=0, y=0)$ of Fig. 9. The slip plane is horizontal. The arrows show the direction and strength of the force acting on the second partial at the arrow position. The forces acting on the dislocations tend to lead to dissociation on the slip plane if no climb forces are present. However, once one of the partials has undergone climb, the force is directed away from the slip plane. Under these conditions the elastic forces drive the partials into the stable 'climb configuration' where both partials are placed vertically above each other.

One might ask whether dissociation by climb is kinetically possible since climb requires the diffusion of all three atom species, O, Sr, and Ti. Diffusion activation enthalpies in the bulk are $1 \mathrm{eV}$ for oxygen and $3.5-4 \mathrm{eV}$ for $\mathrm{Sr}[11,12]$. For $\mathrm{Ti}$ no reliable data exist, but it is assumed that the diffusion of $\mathrm{Ti}$ is the slowest among the three species [12]. It is important to note that for 'climb dissociation' diffusion is required only between the partials (i.e. on the nanometre scale) which means that only few atom jumps are necessary. Furthermore, in the core region of the dislocation diffusion barriers are certainly shallower than in the bulk, i.e. the mentioned activation enthalpies are upper limits. This is probably the reason why Rhodes and Kingery [13] found an activation energy for dislocation pipe diffusion of Ti of only 4.9 $\mathrm{eV}$. These numbers indicate that, under the assumption that core diffusion governs the kinetics of the climb process within Regime $\mathrm{C}$, all three species should be able to migrate over distances required to make climb dissociation possible. Therefore we assume that $a<110>$ 
edge dislocations are dissociated by climb over their whole length within Regime C (Fig. $10 b)$.

Fig. 3 was obtained after a large-angle tilt with the projection of the straight edge dislocations as tilt axis. Their curvature shows that the dislocations have moved in a plane different from their slip plane (Fig.10c).

[Figure 10 about here]

This means that they have undergone climb. The straight appearance of the dislocations when observed perpendicular to the glide plane (Fig. 2) shows that glide appears to be inhibited, most likely because of the possible 'climb dissociation' mentioned above. For glide the partials would have to recombine, thus removing the APB between them, or the APB would have to be dragged along with the partials which would lead to the formation of small dislocation loops. Such loops were indeed observed (see Fig.22 in Ref. [3]) but only very sparsely in some of the TEM samples. In most areas, like those shown in Figs.2 and 3, such structures are not present. Therefore we assume that the dissociated dislocation remains in its elastically stable climb-dissociated configuration and as such undergoes climb.

Macroscopic deformation governed by this climb process needs considerable thermal activation because long-range diffusion of all three atom species, $\mathrm{Ti}, \mathrm{O}$, and $\mathrm{Sr}$ is required. The large activation enthalpies for cation migration (at least $3.5 \mathrm{eV}$ for $\mathrm{Sr}$ and even more for $\mathrm{Ti}[11,12])$ make strong thermal activation necessary for cation diffusion to take place. This is reflected in the strong temperature dependence of the flow stress within Regime C. According to Taeri et al. [3] the activation energy for dislocation movement in Regime $\mathrm{C}$ is about $6 \mathrm{eV}$ for compression along $<100>$. This is higher than the activation enthalpy for diffusion of $\mathrm{Sr}$ and $\mathrm{O}$, but it might be of a similar magnitude as the Ti diffusion enthalpy. It should be mentioned that extremely high diffusion enthalpies for Ti were reported $(>10 \mathrm{eV})$ [14] which would be way too high to make Ti diffusion possible within Regime C. However, Gömann et al. [15] found evidence for Ti migration via $\mathrm{Sr}$ vacancies, and $\mathrm{Sr}$ vacancies are much easier to form than $\mathrm{Ti}$ vacancies. Ti diffusion via $\mathrm{Sr}$ vacancies would, however, lead to some chemical disorder in the wake of the climbed dislocation. How this would influence the dislocation mobility is not clear presently.

Wang et al. [16] performed extensive creep studies of $<100>$ - and $<110>$-oriented $\mathrm{SrTiO}_{3}$ single crystals. They found similar dislocation arrangements as presented in this paper. From the straight appearance of $a<110>$ edge dislocations when observed perpendicular to the slip 


\subsection{Regime A}

plane they concluded that these dislocations move by glide. The present study shows that this interpretation is erroneous.

\subsection{Regime B}

Within Regime B specimens fracture at a fracture stress which strongly increases as temperature increases. The material shatters into small pieces indicating that cracks nucleate in the interior of the material. The formation of such cracks requires high internal stresses. To explain this we assume that the climb dissociation described above also occurs within Regime $\mathrm{B}$, however, because of the lower temperature, only locally along short dislocation segments (Fig. 10a). Unlike in Regime C, these short segments are sessile in Regime B, i.e. only the glissile segments in between are able to move by bowing out under the applied stress (Fig.11). The movement of these glissile segments will soon be retarded by the formation of new sessile segments. This means that long-range motion of dislocations is not possible in Regime B which is reflected in the absence of plastic yielding. A further consequence is that dislocations will not be able to move long distances from the dislocation sources, thus forming obstacles for dislocations emitted from the same source later on. We suppose that the resulting pile-ups lead to sufficiently high internal stresses to initiate crack formation.

[Figure 11 about here]

Increasing temperature leads to shorter mobile segments for which the Orowan stress, which is required for movement, becomes higher. This would explain the increasing fracture stress with increasing temperature. Presently it is unclear how the high internal stresses develop. A possible mechanism would be the pile-up of dislocation loops emanating from the mobile dislocation segments, at obstacles. The dislocation configuration within Regime B is very complicated, showing isolated bundles of dislocations of various Burgers vectors (see Ref. $[3])$. 
At the high-temperature end of Regime A edge dislocations dominate, as it is the case in Regime C. This might be related to the beginning formation of sessile dislocation segments on the edge components disabling the edge segments from changing their line orientation. However, at lower temperatures this will not be possible any more because of the lack of thermal activation. Therefore the dislocations remain on their slip plane and dislocation dissociation is possible only on this plane. As shown in Fig. 4, room temperature deformation leads to the formation of dislocations of various types and deformation at $115 \mathrm{~K}$ results predominantly in screw-type dislocations. For this reason it is likely that towards low temperatures plasticity is governed by screw dislocations. The increase of the flow stress towards lower temperatures and the abundance of screw dislocations can then be interpreted as a decrease of the screw dislocation mobility. Within the Peierls model this could be explained by a high Peierls potential which may originate simply from the strong (partially ionic) bonds in $\mathrm{SrTiO}_{3}$ or from the particular core structure of screw dislocations. The latter would resemble the situation in bcc metals where the core of $a<111>$ screw dislocations dissociates in a non-planar threefold manner which makes thermal activation necessary to make the dislocation mobile. In the case of the $a[0 \overline{1} 1]$ screw dislocation in $\mathrm{SrTiO}_{3}$ the core symmetry is two-fold according to the crystal symmetry. The possible low-index dissociation planes are (100), (011), (111), and (111). Especially for the case of a dissociation on the two $\{111\}$ planes a sessile non-planar configuration of the core could be envisaged. Maybe it is worth noting that we indeed observed a non-planar core configuration of the $a<100>$ screw dislocation in $\mathrm{SrTiO}_{3}$ using HRTEM [7]. We found that the displacement field is more pronounced along $<110>$ directions than along $<100>$ directions. A similar study for $a<110>$ dislocations remains to be done.

The observed screw-type dislocations occur as dislocation dipoles. Apparently the dipole configuration is rather stable. At sites marked by white arrows in Fig.6 the dipoles change between two stable configurations. This leads to the variation of the inside/outside contrast along the dislocation line. The partially vanishing contrast of the screw dipoles is probably due to the local constriction of the two screws, leading to the annihilation of the dipole. For screw dipoles this should be easily achievable if glide on the plane connecting the two dislocations is possible. This connecting plane is the (100) plane in Fig.6. After the constriction elongated dislocation loops are left behind which have short edge components at their two ends. The partial bending or even rotation of the loops indicates that their energetically preferred plane is not the (100) plane. 


\section{Conclusion}

We have performed extensive TEM studies of $\mathrm{SrTiO}_{3}$ single crystals plastically deformed by compression along the $<100>$ direction. The main results are:

(i) In the temperature range from $100 \mathrm{~K}$ to $1000 \mathrm{~K}$ we found almost exclusively dislocations with Burgers vector $a<110>$. However, there is a clear tendency from screw-type to edge-type as temperature increases. The straight appearance of screw dislocations at low temperatures indicates low mobility the reason of which remains speculative at present. We assume that the particular core structure of these dislocations makes them sessile as temperature decreases. This might be possible to be revealed by high-resolution TEM, in a similar way as was done for the bcc metal Mo [18].

(ii) We have shown clear evidence for dislocation climb at high temperatures. The possibility of this process is supported by the similar magnitude of the activation enthalpies for dislocation motion and of cation diffusion.

(iii) A model, based on the climb dissociation of $a<110>$ edge dislocations, was proposed to explain the pronounced DBDT of this perovskite material.

It is still puzzling why dissociation of dislocations in Regime A could not be observed up to now by the weak-beam method. The dissociation width must be below $2 \mathrm{~nm}$ indicating a high APB energy on the $\{110\}$ plane. The knowledge of planar-fault energies $\gamma$ (the ' $\gamma$ surface') in $\mathrm{SrTiO}_{3}$ is clearly required in order to gain further insight into the dissociation mechanisms. Hopefully this can be achieved soon by the help of ab initio calculations. 


\section{Acknowledgements}

We are indebted to U. Salzberger and M. Sycha for the excellent TEM specimen preparation. Discussions with Prof. V. Vitek and A. Heuer are gratefully acknowledged. We thank the German Science Foundation for financial support of C.S. in the framework of the Research Training Group GRK 285. We thank the Referees for very helpful comments which allowed us to improve the paper. 


\section{References}

[1] D. Brunner, S. Taeri-Baghbadrani, W. Sigle, M. Rühle, J. Am. Ceram. Soc. 841161 (2001).

[2] P. Gumbsch, S. Taeri-Baghbadrani, D. Brunner, W. Sigle, M. Rühle, Phys. Rev. Lett. 87085505 (2001).

[3] S. Taeri, D. Brunner, W. Sigle, M. Rühle, Z. Metallkd. 95433 (2004)

[4] D. Brunner, private communication

[5] Z. Zhang, W. Sigle, M. Rühle, Phys. Rev. B66 094108 (2002)

[6] Z. Zhang, W. Sigle, W. Kurtz, M. Rühle, Phys. Rev. B66 214112 (2002)

[7] Z. Zhang, W. Sigle, W. Kurtz, Phys. Rev. B69 144103 (2004)

[8] R. A. de Souza, J. Fleig, J. Maier, O. Kienzle, Z.L. Zhang, , W. Sigle, M. Rühle J. Am. Ceram. Soc. 86, No.6, 922 (2003)

[9] T.E. Mitchell, K.P.D. Lagerlöf, A.H. Heuer, Mater. Sci. Techn. 1944 (1985)

[10] Calculated by a computer code written by Prof. V. Vitek.

[11] R. Meyer, R. Waser, J. Helmbold, G. Borchardt, Phys. Rev. Lett. 90105901 (2003)

[12] K. Gömann, G. Borchardt, M. Schulz, A. Gömann, W. Maus-Friedrichs, B. Lesage, O. Kaïtasov, S. Hoffmann-Eifert, T. Schneller, Phys. Chem. Chem. Phys. 72053 (2005)

[13] W.H. Rhodes, W.D. Kingery, J. Am. Ceram. Soc. 49521 (1966)

[14] M.J. Akhtar, Z. Akhtar, R.A. Jackson, J. Am. Ceram. Soc. 78421 (1995)

[15] K. Gömann, G. Borchardt, A. Gunhold, W. Maus-Friedrichs, H. Baumann, Phys. Chem. Chem. Phys. 63639 (2004).

[16] Z. Wang, S.-I. Karato, K. Fujino, Phys. Earth Planet. Inter. 79299 (1993).

[17] T. Matsunaga, H. Saka, Phil. Mag. Lett. 80597 (2000).

[18] W. Sigle, Phil. Mag. 791009 (1999). 
Figure captions

Fig.1: Temperature dependence of the flow stress, $\tau_{\mathrm{o}}$, and fracture stress, $\tau_{\mathrm{fr}}$, in $\mathrm{SrTiO}_{3}$ under compressive loading along different crystallographic axes. Three different regimes can be distinguished: Regime A at low temperatures where the flow stress is low but increasing towards low temperatures, Regime $\mathrm{B}$ where the material fails catastrophically at a temperature-dependent fracture stress, and Regime $\mathrm{C}$ where a strongly temperature-dependent flow stress is found. The classification labels (A, B, C) shown in the upper part of the Figure are for the $<100>$ compression axis, whereas those shown in the lower part $(a, b, c)$ are for orientations other than $<110\rangle$. The low-temperature flow stresses coincide for all orientations, but the temperature range of Regime B and the high-temperature flow stresses are orientationdependent. This is related with the different glide systems: The easy glide system $a<100>\{100\}$ which is active for non $-<100>$ compression in Regime $\mathrm{C}$ cannot operate with a load parallel to $<100>$ because of the vanishing Schmid factor.

Fig.2: Bright-field image of the dislocation configuration after deformation in Regime C. The incident beam direction is close to [233]. Most of the observed dislocations are straight, running from top left to bottom right, and are of edge type with Burgers vector $a[01 \overline{1}]$. The other dislocations (vertical line direction and from top right to bottom left) also have $a<110>$ Burgers vectors but are no pure edge dislocations (for details see Reference [3]). They belong to other $a<110>\{110\}$ slip systems which are all activated by compression along $<100>$.

Fig.3: Bright-field image of the same area as Fig.1 but tilted by $52^{\circ}$. The observation direction is close to [001]. The straight dislocations in Fig. 1 are now clearly curved. For clarity some corresponding dislocations are marked by letters. The double contrast of dislocations marked by asterisks is because $g \cdot b$ is equal to 2 whereas the product is unity for the other dislocations.

Fig.4: Weak-beam image of the dislocation configuration after deformation at room temperature. The dislocations have various line directions with a slight bias towards the screw orientation (parallel to $g$ ). 
Fig.5: Weak-beam image of the dislocation configuration after deformation at $115 \mathrm{~K}$. Unlike the dislocation pattern at room temperature, here dislocations are mostly of pure screw type and few of them of pure edge type.

Fig.6: Weak-beam images taken under $+\boldsymbol{g}$ - and $-\boldsymbol{g}$-conditions showing pronounced inside/outside contrast. The image is an enlarged part of Fig. 5. The double contrast is not always continuous along the dislocation line. At some places double contrast is visible at $+\boldsymbol{g}$, whereas at other places $\boldsymbol{- g}$ creates double contrast (marked by arrows).

Fig.7: Weak-beam image of screw dislocations showing a variation of the contrast along their dislocation line. One location where the contrast has vanished is marked by an asterisk. The dipoles appear to be decomposed into dislocation loops. The loops appear to be slightly inclined with respect to the general direction of the dislocation line. The image is an enlarged part of Fig. 5.

Fig.8: HRTEM image of an $a<110>$ edge dislocations dissociated by climb. This image is taken from a bicrystal specimen containing a low-angle $<110>$ tilt grain boundary [6].

Fig.9: Plot of the forces acting between two $a / 2<110>$ partials. One of the partials is in the centre $(x=0, y=0)$. The slip plane is horizontal. The arrows show the direction and strength of the force acting on the second partial at the arrow position.

Fig.10: Sketch of the possible structure of the $a<110>$ edge dislocation in Regimes B and C. Two adjacent $\{110\}$ planes are shown in grey. The non-dissociated dislocation is located on the upper plane. (a) The dislocation is dissociated by climb along a short segment. (b) The dislocation is dissociated along its whole length. (c) The dislocation has moved out of the slip plane by climb, i.e. perpendicular to the slip plane.

Fig.11: Sketch of the $a<110>$ edge dislocation being dissociated on the left and right side. The non-dissociated part can bow out under an applied stress. 


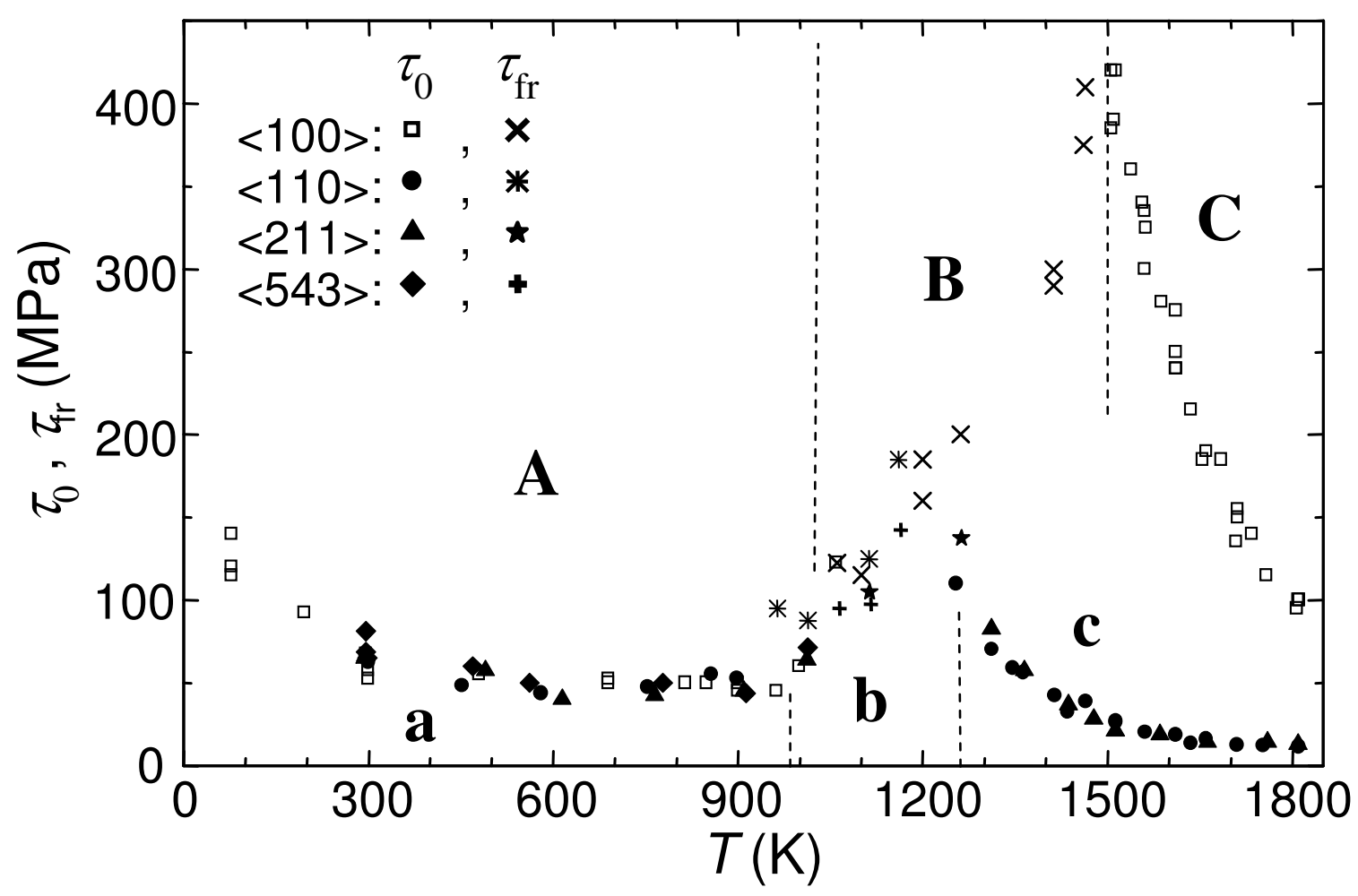

Fig. 1 
Fig.2

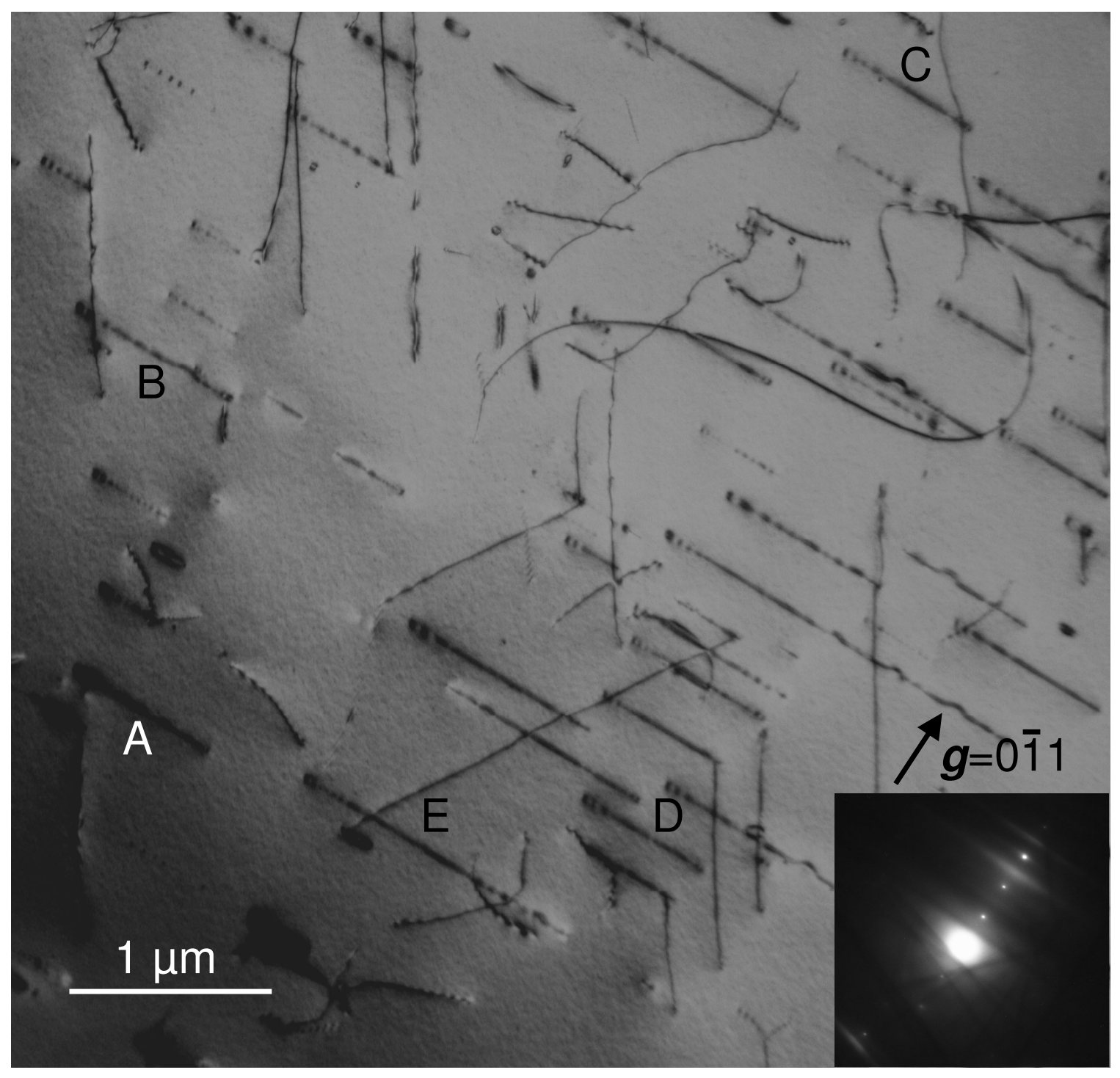

http://mc.manuscriptcentral.com/pm-pml 
Fig.3

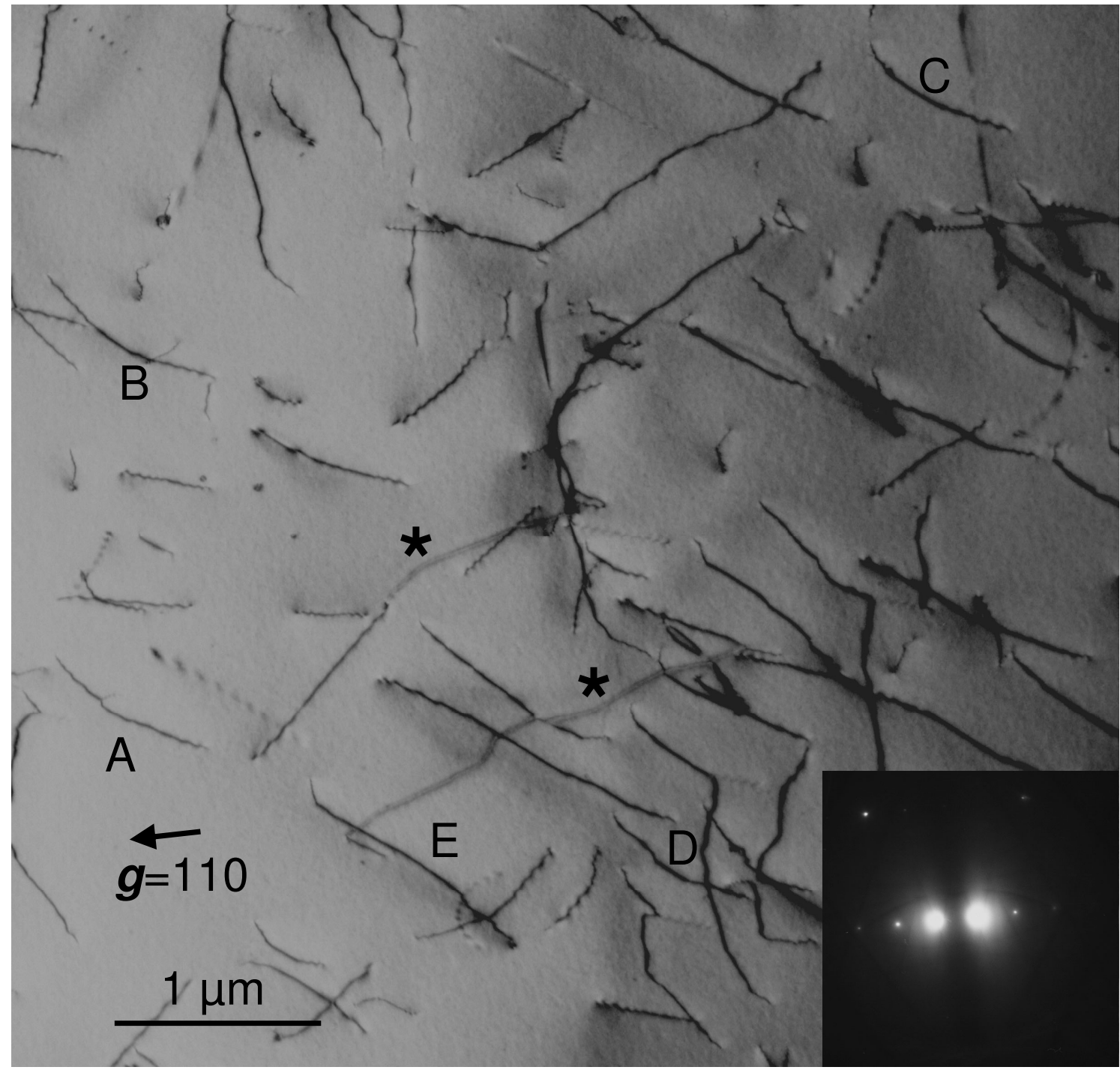

http://mc.manuscriptcentral.com/pm-pml 
Fig. 4

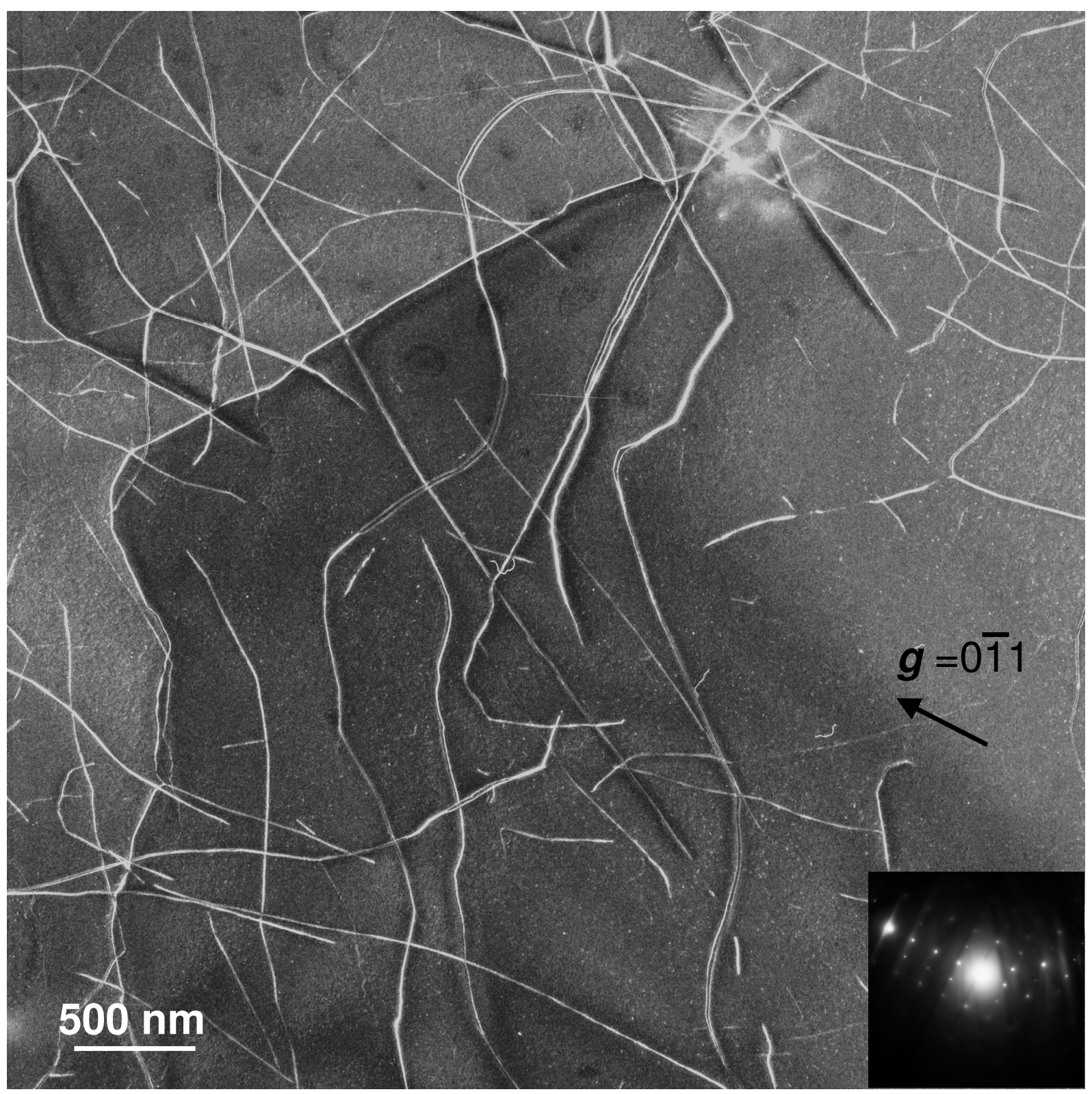

http://mc.manuscriptcentral.com/pm-pml 
Fig.5

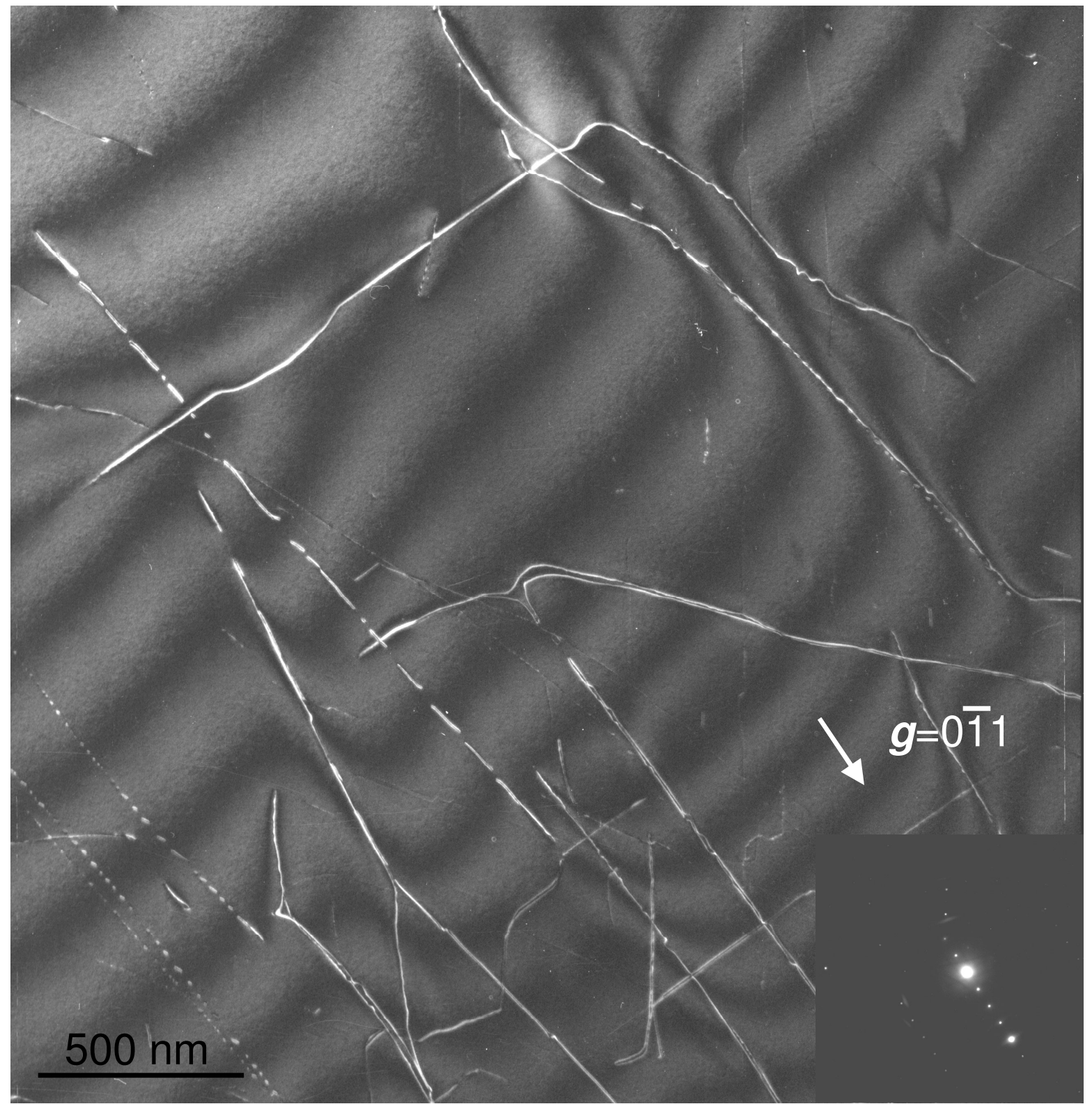


Fig.6
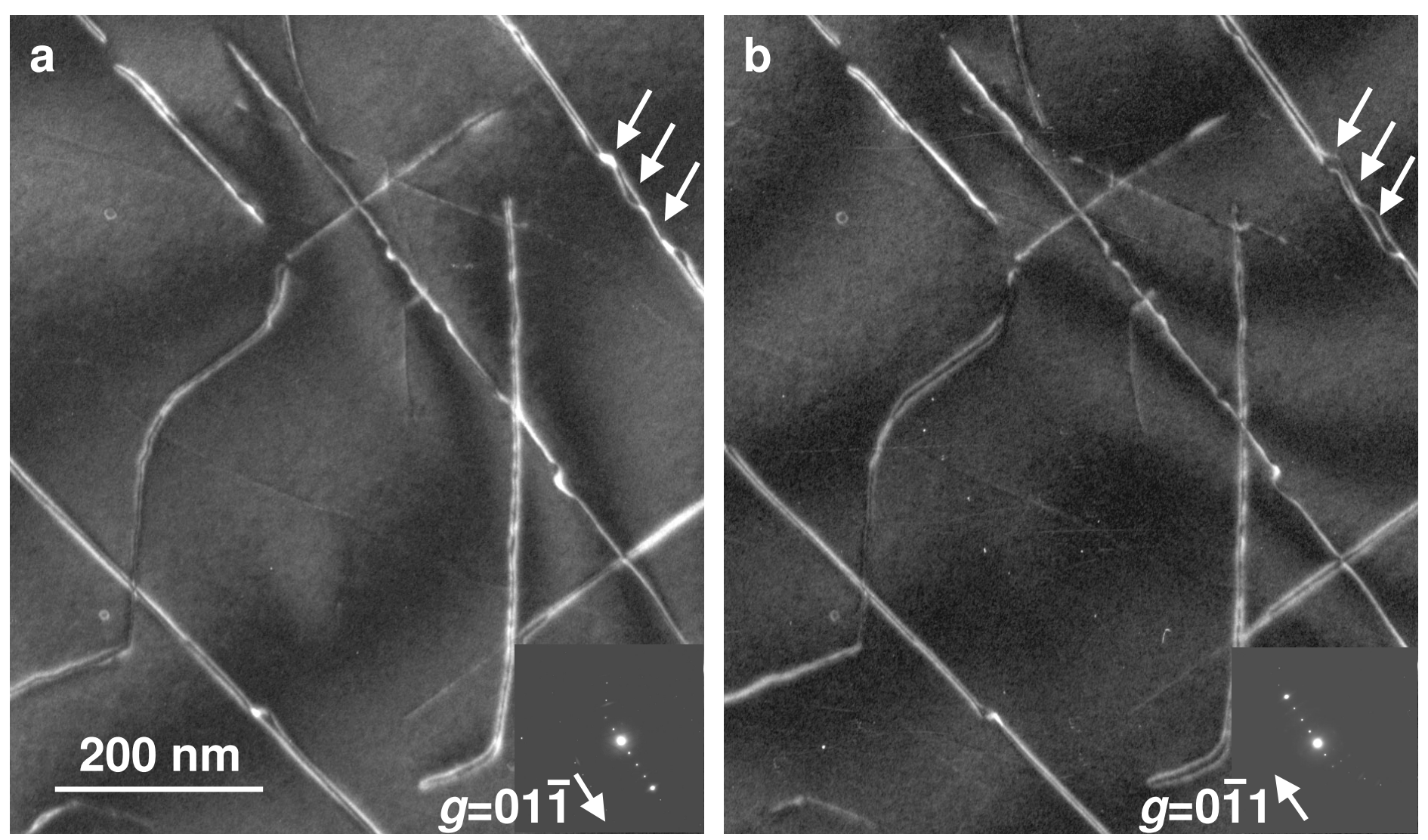

http://mc.manuscriptcentral.com/pm-pml 
Fig.7

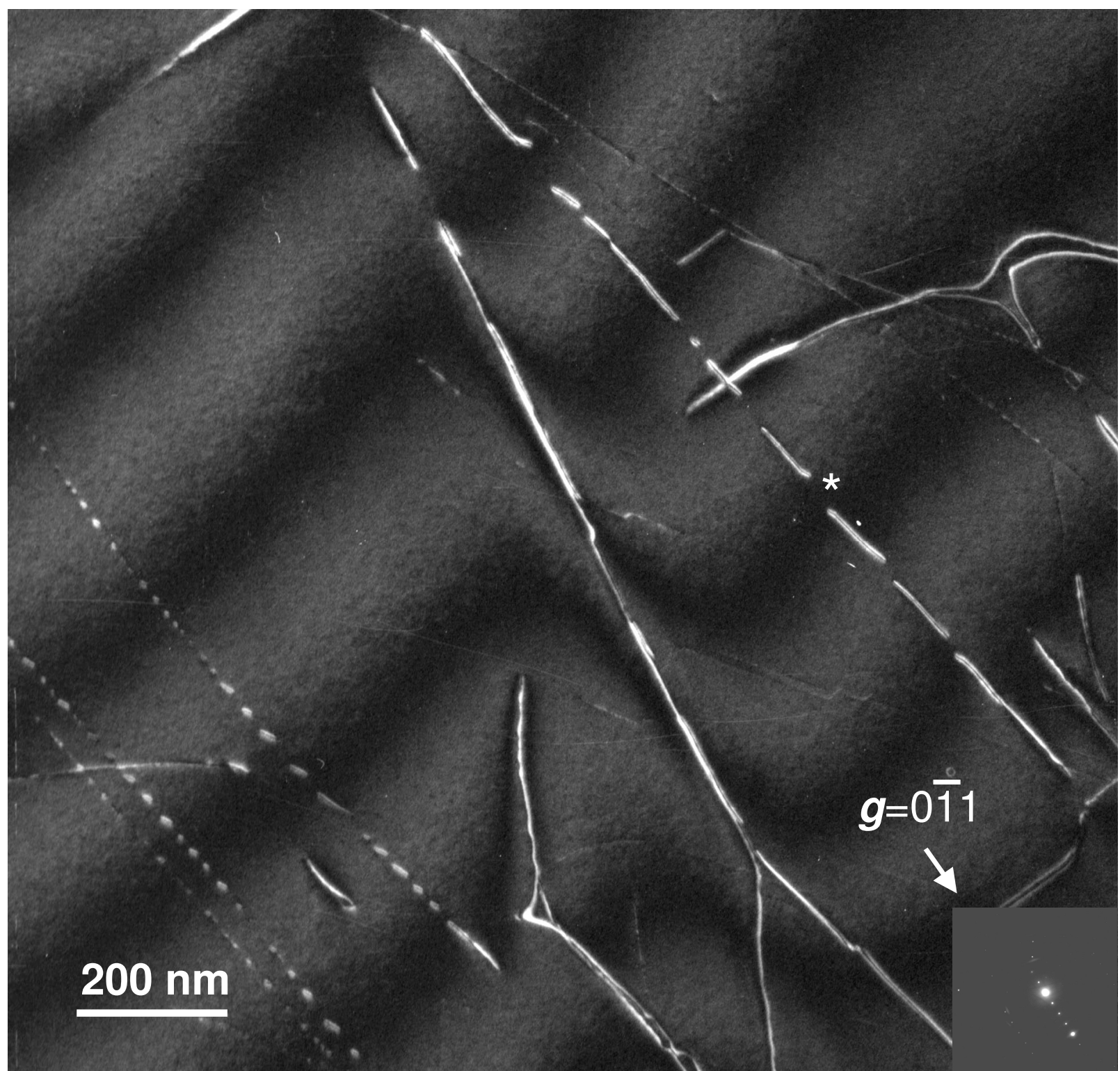

http://mc.manuscriptcentral.com/pm-pml 
1

2

3

6

7

10

11

12

13

14
15

15

16
17

17
18

19

20

21

22

23

25

26

27

28

29

30

31

32

33

34
35

35

36
37

37

38
39

40

40
41

42

43

44

45

46

47

48

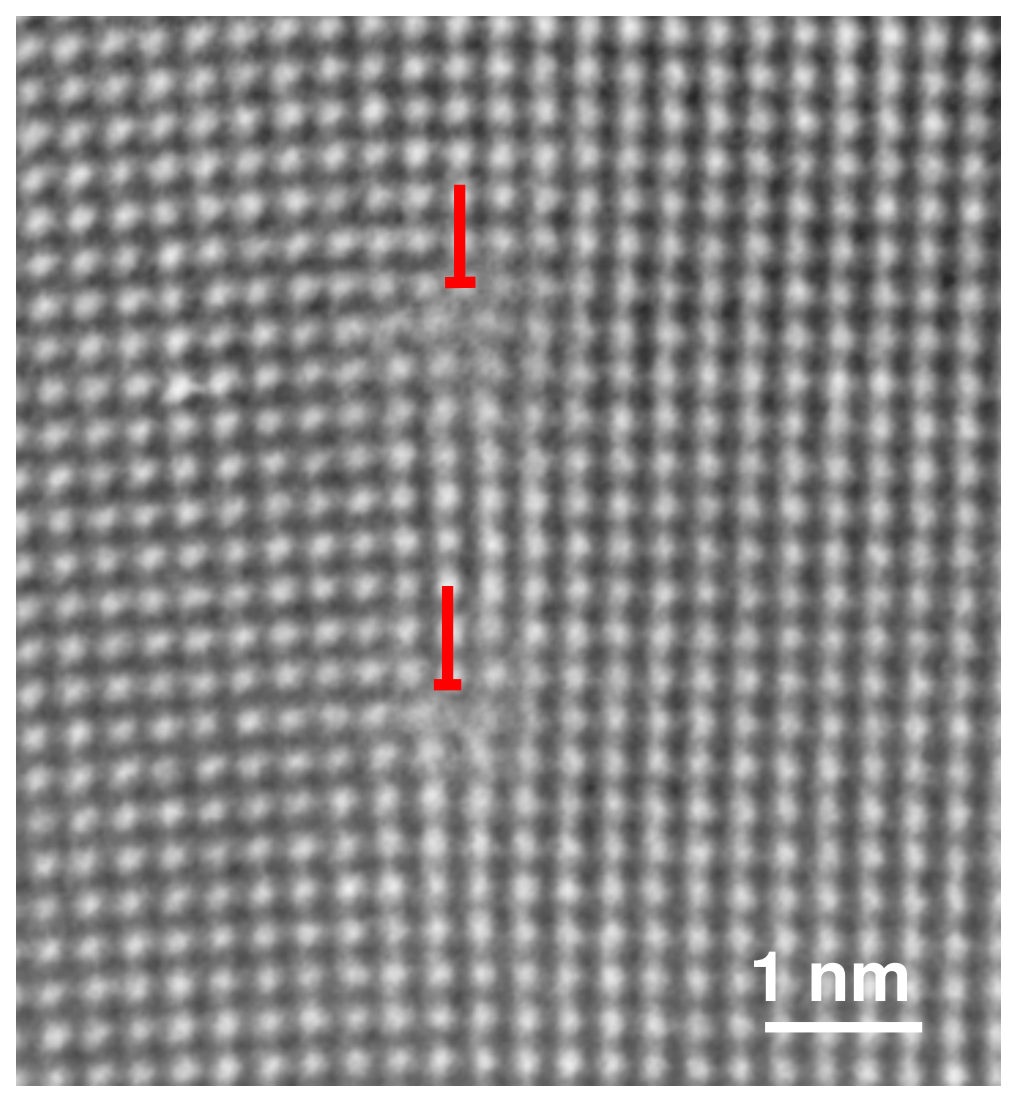

http://mc.manuscriptcentral.com/pm-pml 
Fig.9

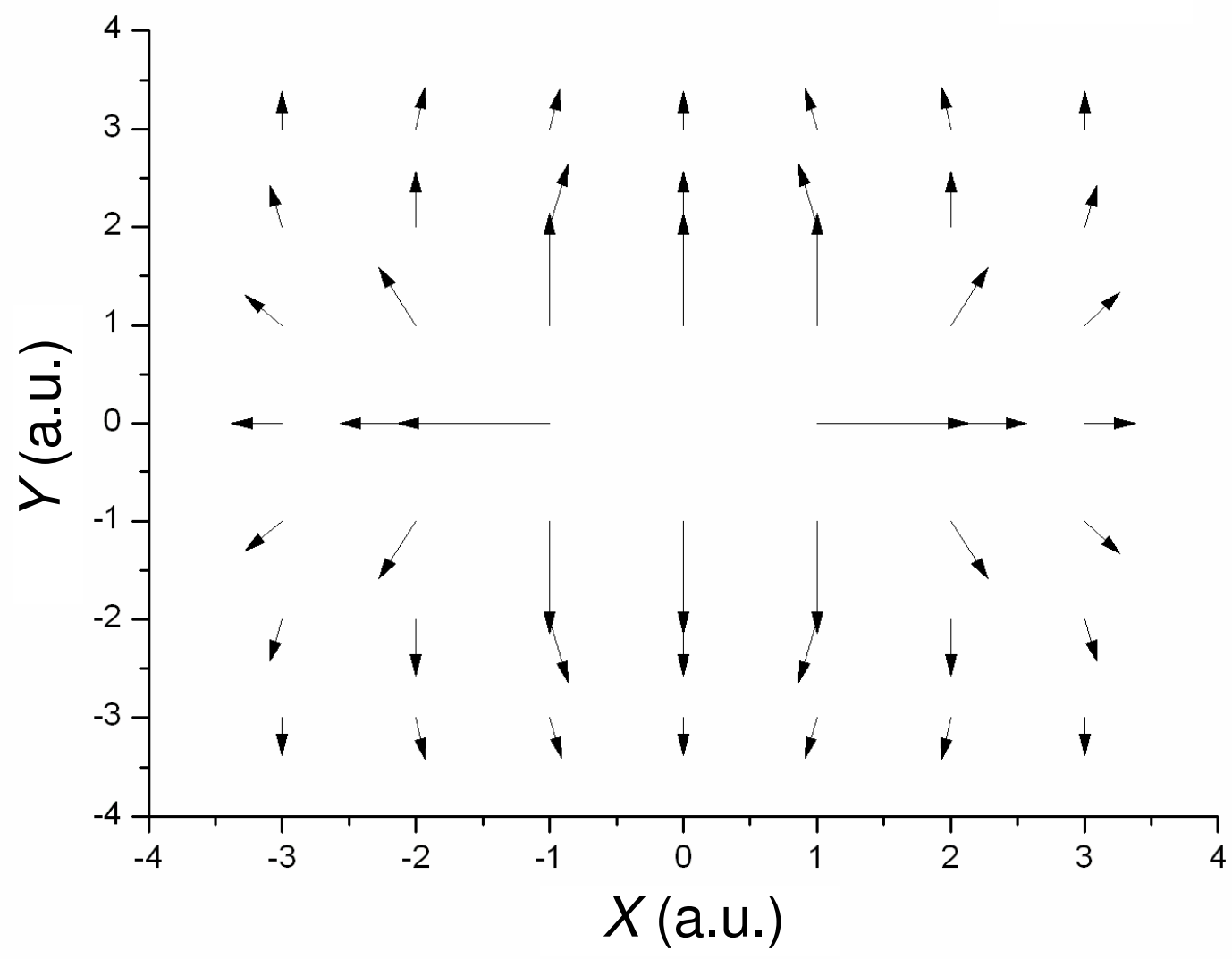

http://mc.manuscriptcentral.com/pm-pml 
Fig.10

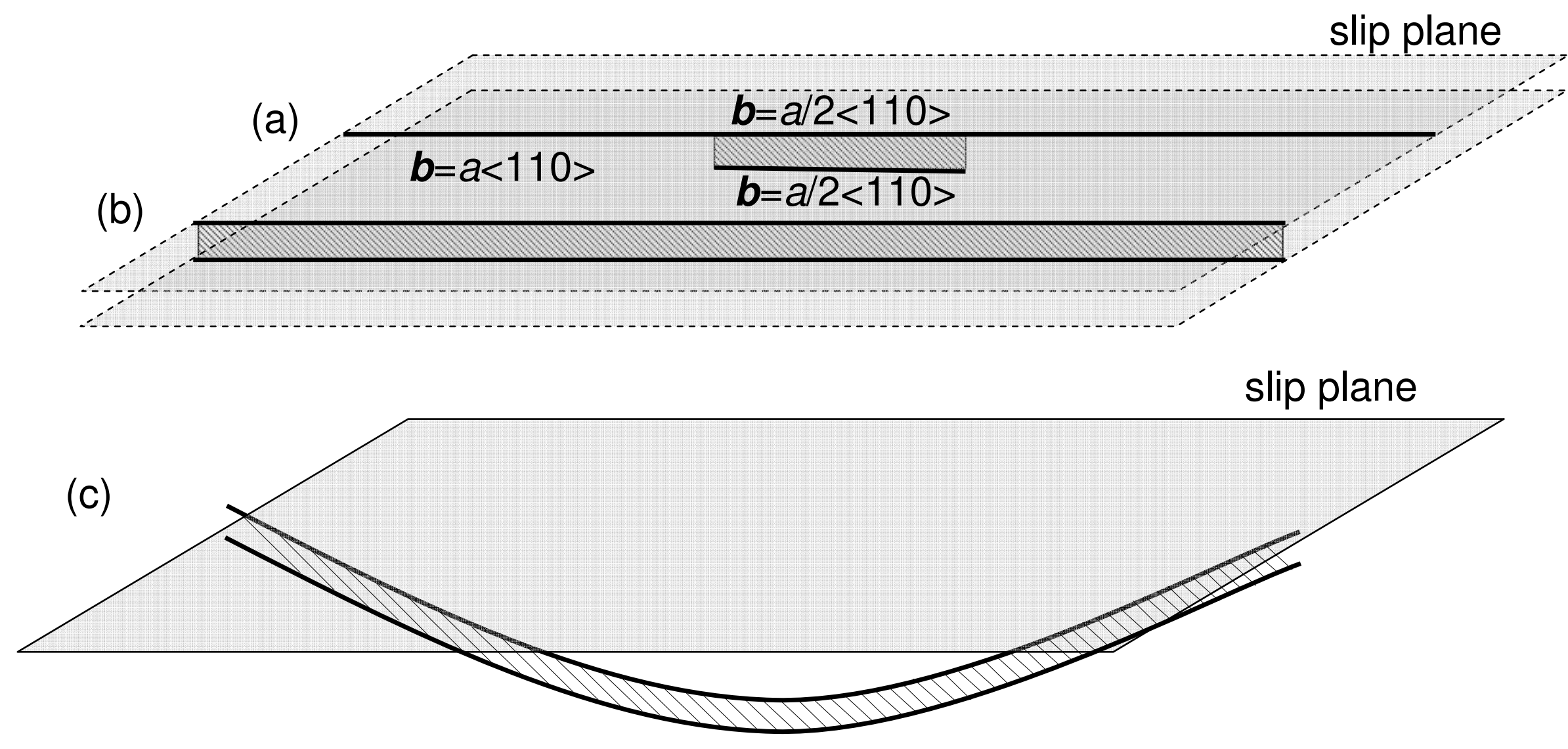


Fig.11

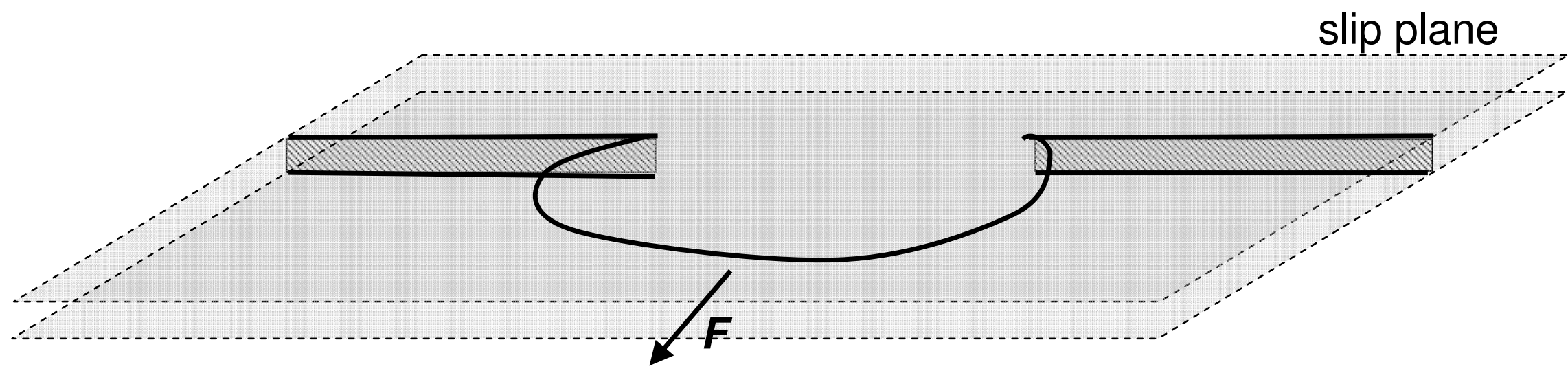

